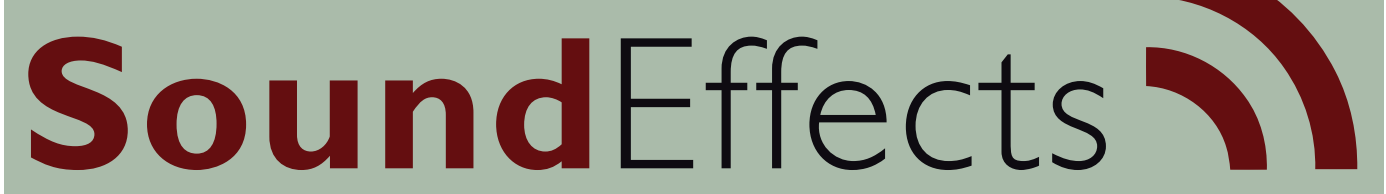

An Interdisciplinary Journal of Sound and Sound Experience

\title{
Solène Marry
}

\section{Ordinary sonic public space}

Sound perception parameters in urban public spaces and sonic representations associated with urban forms

\section{Dr. Solène Marry \\ Research Engineer \\ Urban Institute of Grenoble \\ CREIDD (Center for Research and Interdisciplinary Studies on Sustainable Development) / University of Technology of Troyes solene.marry@utt.fr}




\section{Abstract}

The research referred to in the article concerns the factors influencing the perception of ordinary sonic public space and everyday sounds. Sound perception parameters, such as vegetation or sound sources, are analysed in urban public spaces. This research, which is based on my PhD project, tries to understand how urban people perceive their sonic environment and try to contribute to sonic ambiance knowledge. The research is based on a qualitative investigation conducted among 29 people. It is, on the one hand, based on questionnaires and focus groups in situ and, on the other hand, on individual interviews (in-depth interviews, sonic mind maps), and it illustrates different parameters (temporal, spatial, sensitive and individual) that influence a person's assessment of the sound environment. This qualitative investigation is correlated with acoustic measures in two seasons. The results show, among other things, the impact of vegetation and urban fittings on sonic perception, and they underline the influence of city planning and urban fittings on sound perception in public urban spaces.

Keywords: sound perception, public squares, urban sounds, sonic representations, urban forms

\section{Introduction}

This article presents different tools for assessing soundscapes within urban public spaces: more precisely, three public squares with various forms and urban morphologies of the surrounding districts: Place des Tilleuls in the old, dense and continuous (unbroken urban fabric) centre; Place Mistral-Eaux-Claires in the dense, but not continuous district named Eaux-Claires; and Place Centrale located on campus, not dense and discontinuous.

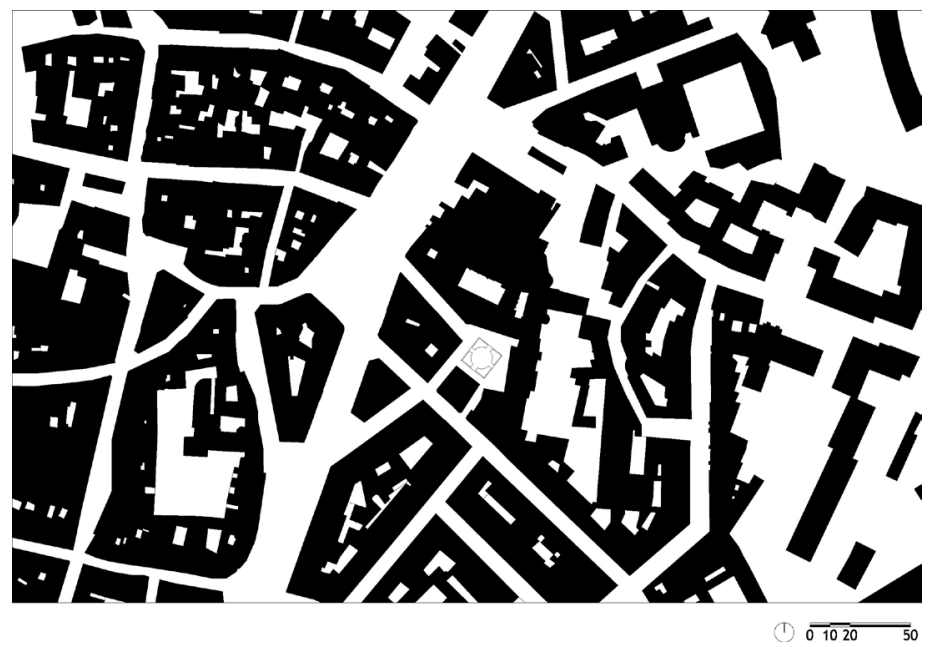

Figure 1: Place des Tilleuls, an old dense and continuous centre district 


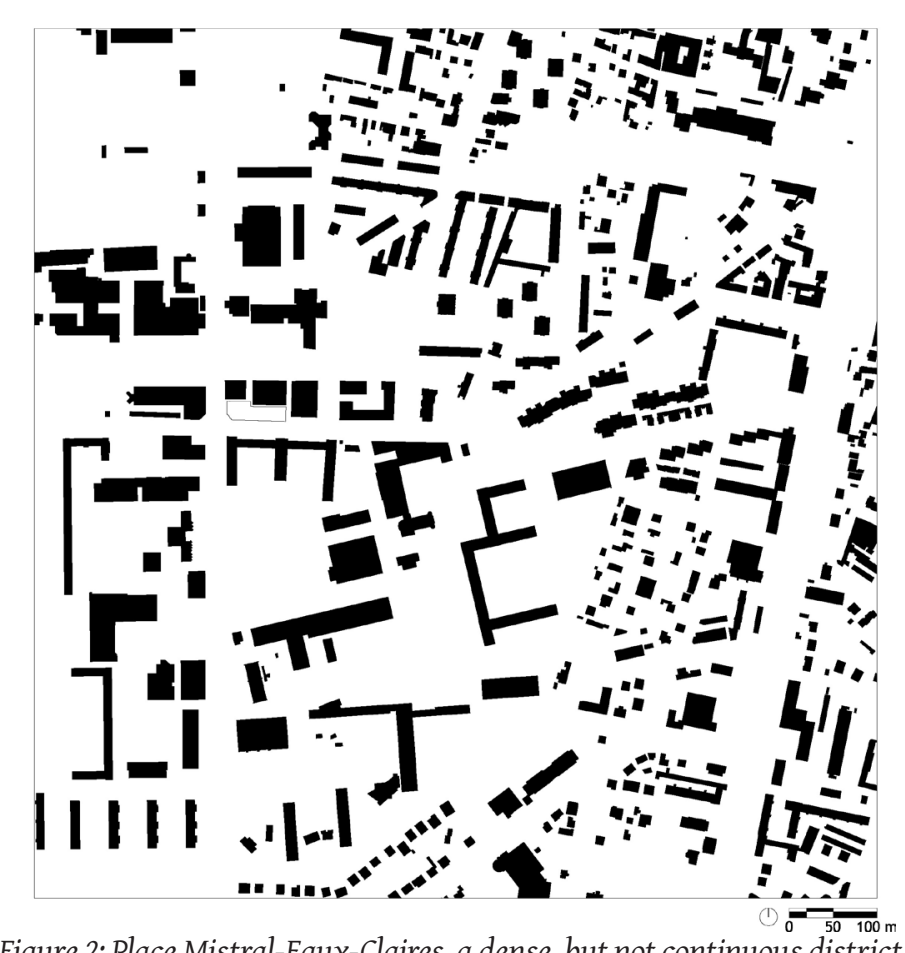

Figure 2: Place Mistral-Eaux-Claires, a dense, but not continuous district

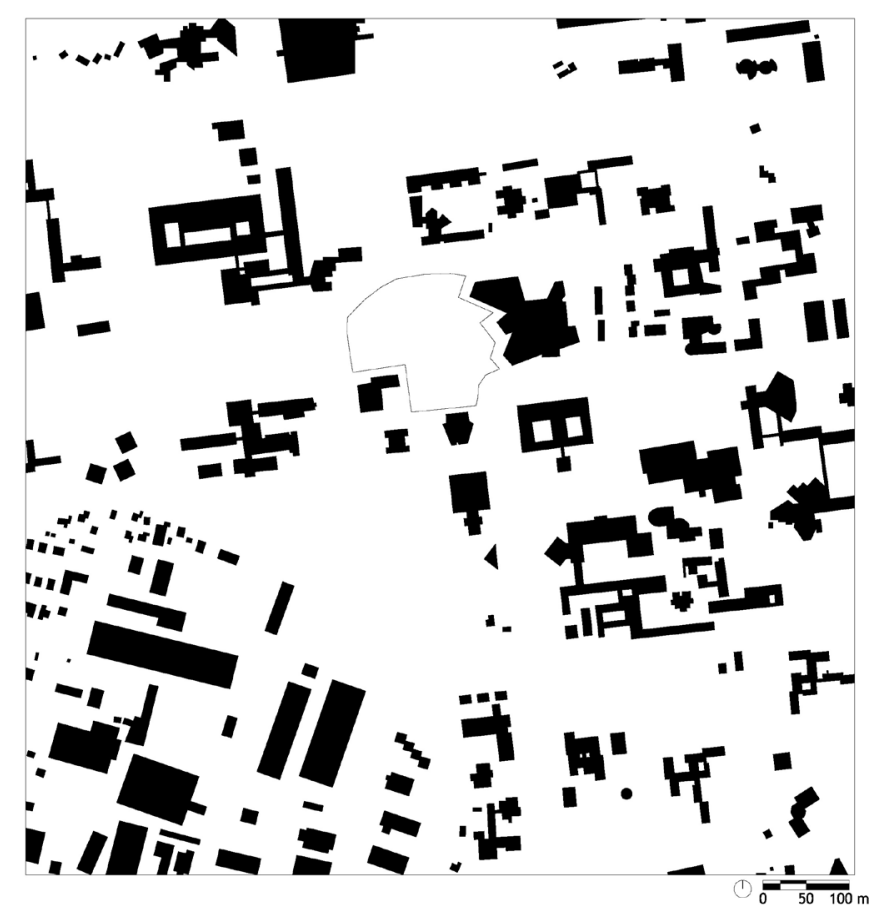

Figure 3: Place Centrale, not dense and discontinuous district 
For this research, the field of study consisted of three urban public squares. In this study, we chose three different types of squares (size, form, gap etc.) shown in figures 4,5 and 6 .

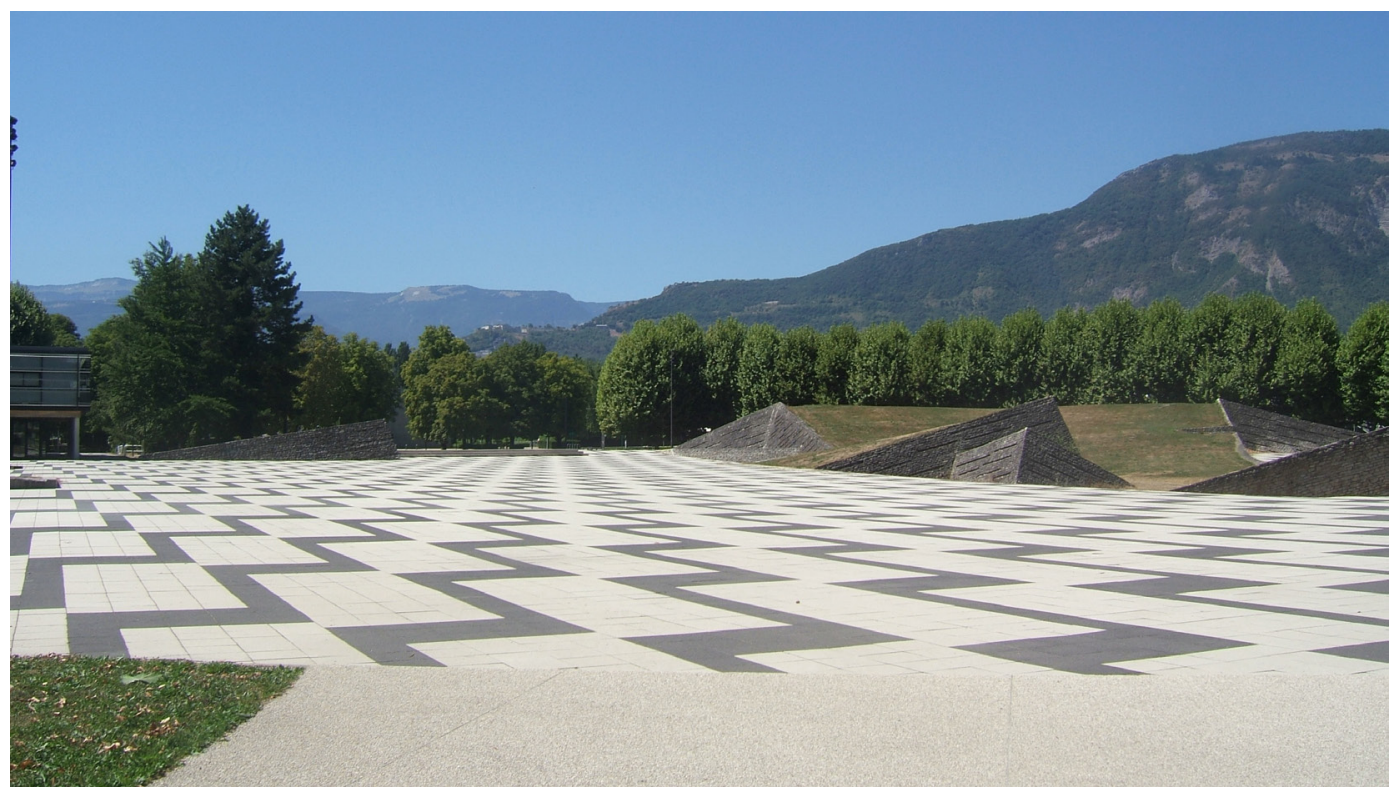

Figure 4: Place Centrale, campus, Saint-Martin-d'Hères

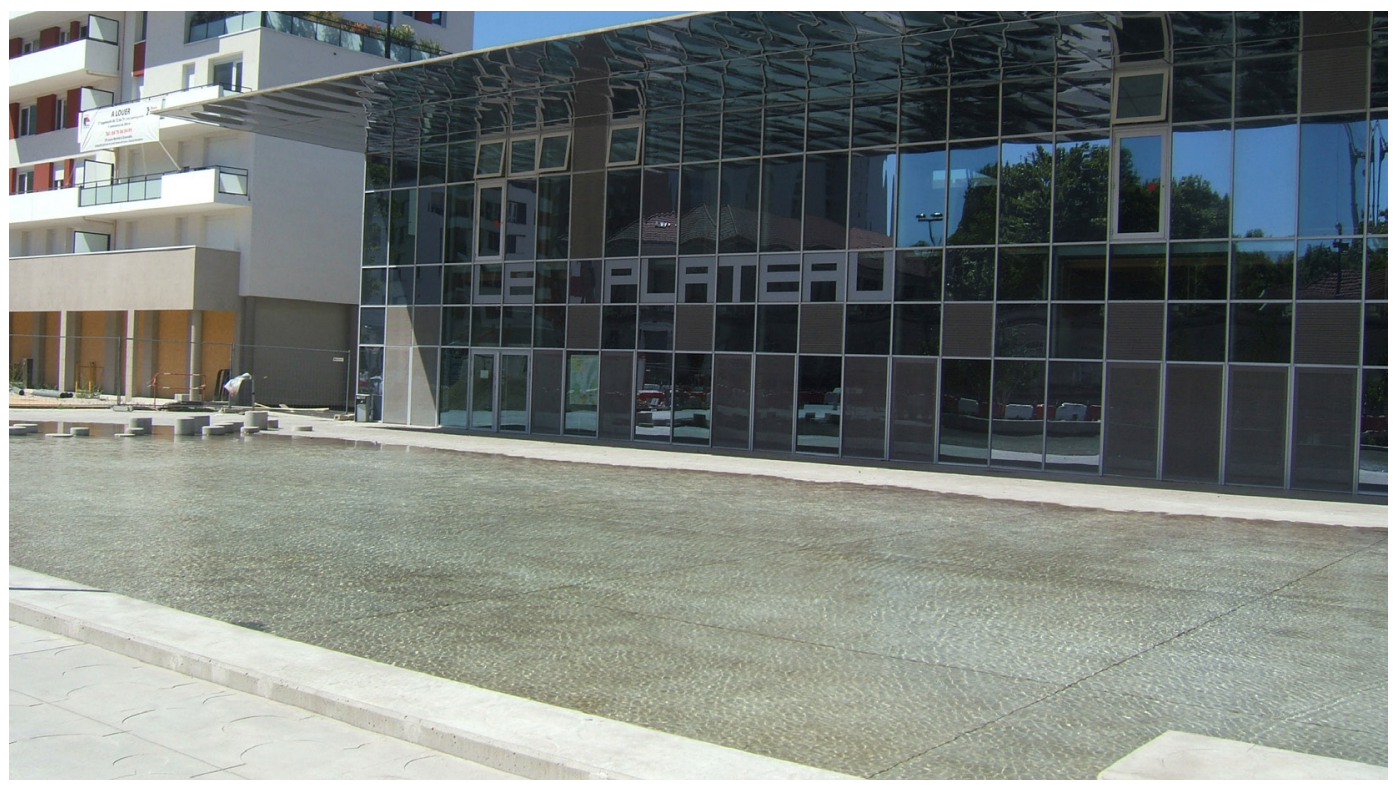

Figure 5: Place Mistral-Eaux-Claires, urban regeneration zone, Grenoble 


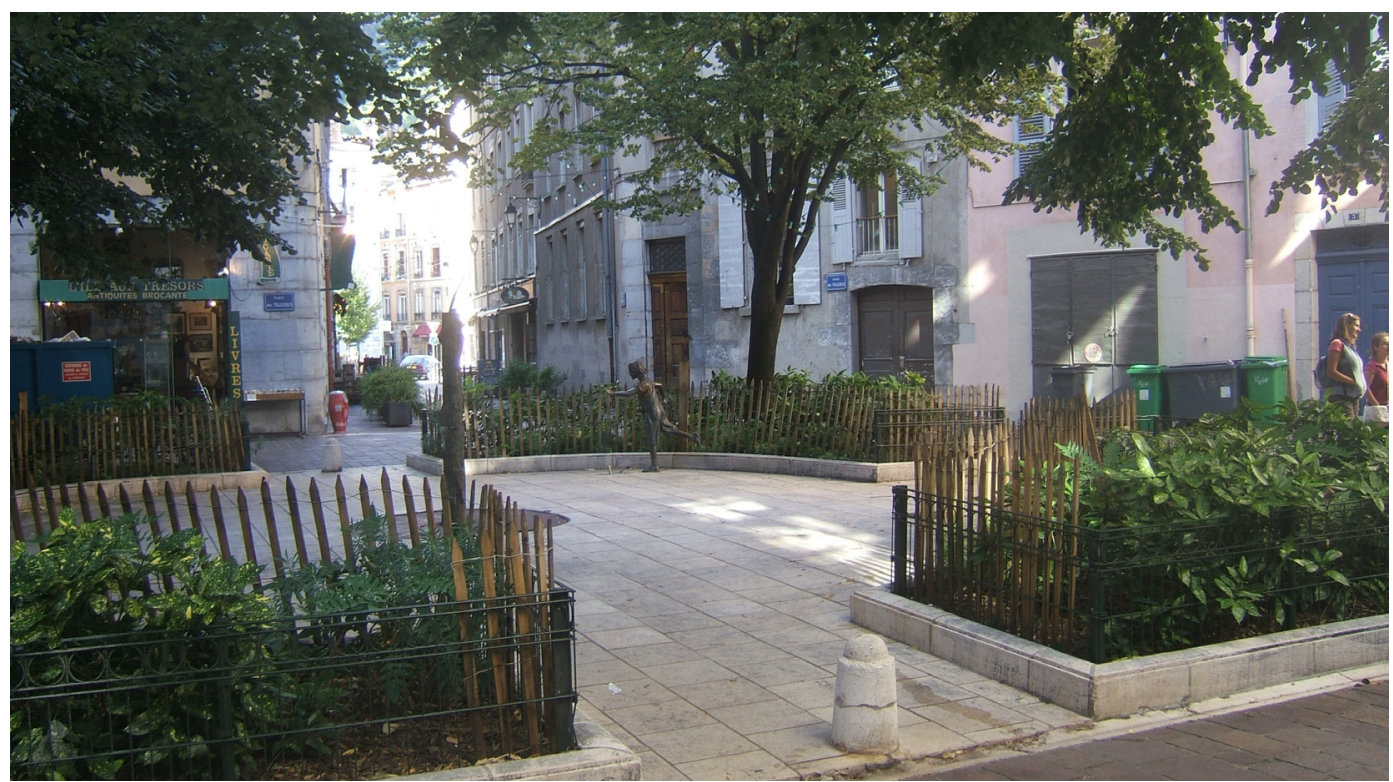

Figure 6: Place des Tilleuls, historical urban center, Grenoble

Raymond Murray Schafer, the Canadian composer, music educator and environmentalist, introduces the notion of soundscape in his reference book The Tuning of the World (Schafer, 1977) and in his work for the World Soundscape Project. This neologism, created by the contraction of the words landscape and sound, illustrates the image of a 'sonic environment'. The introduction of this new concept reflected how the sonic field had progressively come to be considered in the scientific community; the sonic aspect is not only understood as a simple environmental nuisance, but also as a daily sonic quality. Schafer's view, according to which the sounds of postindustrial civilisation and the sounds linked to urbanisation are almost systematically depreciated, is criticised by the sound artist Francisco Lopez. In particular in his essay entitled 'Schizophonia vs l'Objet Sonore' (1997), Lopez questions Schafer's very idea of soundscape harmony, as post-industrial sounds are qualified by Schafer as lo-fi (low fidelity) as opposed to the natural context, hi-fi. Lopez therefore gives the example of the equatorial forest, a dense soundscape where information collides for human sonic perception. Moreover, for Lopez and other contemporaries, industrial sounds have artistic potential. Researchers such as Lopez continue this sonic ecology initiated by Schafer in a comprehensive vision of our daily relation to sound. The research presented in this paper on sonic perception and our daily and ordinary relation to sounds is part of this paradigmatic perspective.

The particularity and challenge of my $\mathrm{PhD}$ research subject lie in the confrontation between the urban vision of the public space, the physical approach to acoustic measurement and the consideration of how the sonic environment is perceived in several types of public spaces. In our study we problematise the understanding and 
assessment of sound perception in order to highlight some of its relevant parameters.

Our research deals with sonic perception in urban environments. Schafer reminds us that "the English term "noise" can etymologically be traced back to the Old French word noyse and to the eleventh-century Provençal words noysa, nosa or nausa, but its origin is uncertain' (Schafer, 1979).

Which parameters influence sonic perception? This question was the guiding force of my investigations, and it is particularly difficult to answer because of the number of determining variables influencing sound percepts. Which impact do the temporalities of a space have on perceived ambiance? Which factors influence this perception the most? Are there interrelations between perceptive criteria? What are sonic percepts the result of? The legitimisation in search of parameters influencing the perception of sonic ambiances in a public space is linked to the scarcity of research in this field.

A square is a particular public space typology, which is at the base of the public space concept (Greek agora). A square also has a clear spatial delimitation and strong mental representations. The spatial representation and localisation of sonic events are particularly interesting for sonic mind map analysis. In his thesis, researcher in psychology, Alain Leobon, who used mind maps, found spatial distortion related to a sonic event interesting. When he noted that "the sonic event "cars" distorts mental representations because of its strong spatial symbolism. [...] even if this space is pedestrian, a car going along in the far distance was enough to draw a street and place it side by side with the square itself' (1986, p. 154).

\section{Elaborate methodological protocol}

The methodology is based on a qualitative investigation and acoustic measurements in order to correlate perceptive and physical data. A preliminary test allowed us to develop the questionnaire and to confirm the field of study. The investigation was conducted in the course of six months from September 2009 to March 2010. This qualitative investigation was split into two different parts: the on-site part was executed during two different seasons with the same participants, and the second part was carried out afterwards and consisted of individual interviews.

S. Duchesne and F. Haegel (2004) emphasise that few studies use both methods (collective and individual interviews) with the same persons. Collective interviews are complementary to individual interviews; it allows us to compare the comments presented in a group and those delivered face to face. Our methodology is quite different from standard collective interviews, because it relies on in situ collective interviews in urban zones. Indeed, laboratory experiments cannot be similar to contextualisation on site, even when they tend to reproduce a specific environ- 
ment. The persons interviewed in the three public spaces are thus characterised by the shared experience of common spaces, which makes it easy to share the same point of view on the present situation (with the questionnaire and the focus group).

The first part of the methodology resulted in 174 on-site questionnaires (each interviewee answered the questionnaire for each season and on each square), 513 photographs (the interviewees were asked to take three photographs on each square for both seasons representing the 'ambiance'), 18 on-site focus groups (together the participants discussed their feelings and evaluation of the square) and 22 acoustic measurements. The interviewees were not informed of the sonic focus of the investigation; it was presented to them as an investigation of general ambiance.

This first collective part of the methodological protocol has been associated with 29 individual in-depth interviews with the same interviewees. These individual interviews included:

- In-depth interviews about their memory of the three squares in particular (ambiance, comfort, environmental sounds) and their urban practices.

- A commentary of the pictures taken on the squares.

- Five cognitive map tests: the interviewees were asked to draw the sonic environment of the three squares, the ideal and the worst sonic ambiance of a public square.

- Ranking urban typologies in association with quality environmental sounds.

Acoustic measurements were achieved in the same seasons as the survey and on the same day of the week in order to compare the same urban temporalities. The overall aim of the methodology was to compare acoustic levels and types of environmental sounds with sound perception. This is why A-weighted equivalent sound levels $\left(\mathrm{L}_{\text {Aeq, 1s }}\right)$ were measured on each square. $22 \mathrm{~L}_{\text {Aeq, 1s }}$ five-minute measurements were done on fixed points and ten-minute measurements while travelling around the square, making a note of the sonic events. Each point has been measured several times. The measurements were not realised at the same time as the qualitative investigation in order to not interfere with the interviewees' answers. The illustrative variables associated with the acoustic measures allow us to identify links with the acoustic indicators, but they are not presented in this article. I will focus on sonic perception, representation and assessment.

\section{Assessment of public spaces}

Assessing an environment is defined by G. Moser and $\mathrm{K}$. Weiss as 'evaluating the individuals' degree of satisfaction and effects in relation to a given environmental unit' (Moser \& Weiss, 2003, p. 88; my translation). This concept of environmental 
assessment is relevant in space sciences and in public space evaluation in particular. One of the tasks urban planners face is to understand and satisfy the expectations of the users of a given space. Therefore, researchers learn how to develop methods to analyse environmental experiences and ensuing spatial assessments. The challenge of these methods lies in the transition from the experience of an in situ space to the assessment hereof.

Among other things, assessing a public space requires an assessment of its sonic space; or, in other words, judgement of the sonic environment is a part of the overall spatial assessment. In this respect, Southworth, in a 1969 pioneer study of this matter, explained the impact of the information underlying sounds, the context they are perceived in and their level in the assessment of an urban environment (in this case, Boston). Moreover, a study based on three Spanish urban spaces shows that the sonic identity of different urban environments has an influence on the interviewees' assessment of these spaces (Lopez-Barrio \& Carles, 1995).

The questionnaires realised during the present investigation were produced with the aim to understand, among other things, the feelings of the people who were interviewed and their judgement of sonic ambiance. In the on-site questionnaire, interviewees were asked to qualify the ambiance of the area as a whole. The results are the words used by all interviewees to describe the general ambiance of the three squares. The sonic ambiance is intrinsically connected to the space; the public space is characterised by an ambiance which varies temporarily. The output of the survey consists of words used by all interviewees to describe the general ambiance of Place Centrale, Place Mistral and Place des Tilleuls. To the question 'How would you describe the ambiance of this square?', the ambiance (43 occurrences) is first described in sonic terms: this ambiance is judged as 'quiet' (52 occurrences in 174 questionnaires).

Regardless of the described square, ambiance is continuously associated with sonic ambiance (figure 7), because for Places Centrale and Tilleuls the lemma 'quiet' is quoted 26 times, and for Place Mistral it is the lemma 'noise' (8 occurrences). Interviewees frequently described the sonic ambiance as follows: 'I feel peace and it is calm. A comforting silence' (Interviewee 22CRT). We can conclude that global ambiance is closely associated with sonic ambiance in an urban public space.

Another observation concerns temporality; the word 'quiet' is used in both seasons (26 occurrences) in order to describe the general ambiance of the squares, but interviewees describe ambiance with more sound-related words ('noise' and 'calm') in the second on-site questionnaire. The lemma 'quiet' is observed as one of the terms characterising the squares 21 times in September and 27 times in December, while the term 'noise' is quoted more frequently in December (11 occurrences) than in September (6 occurrences). 


\section{\#soothing \#tramwayplace \\ welcoming \#mountain}

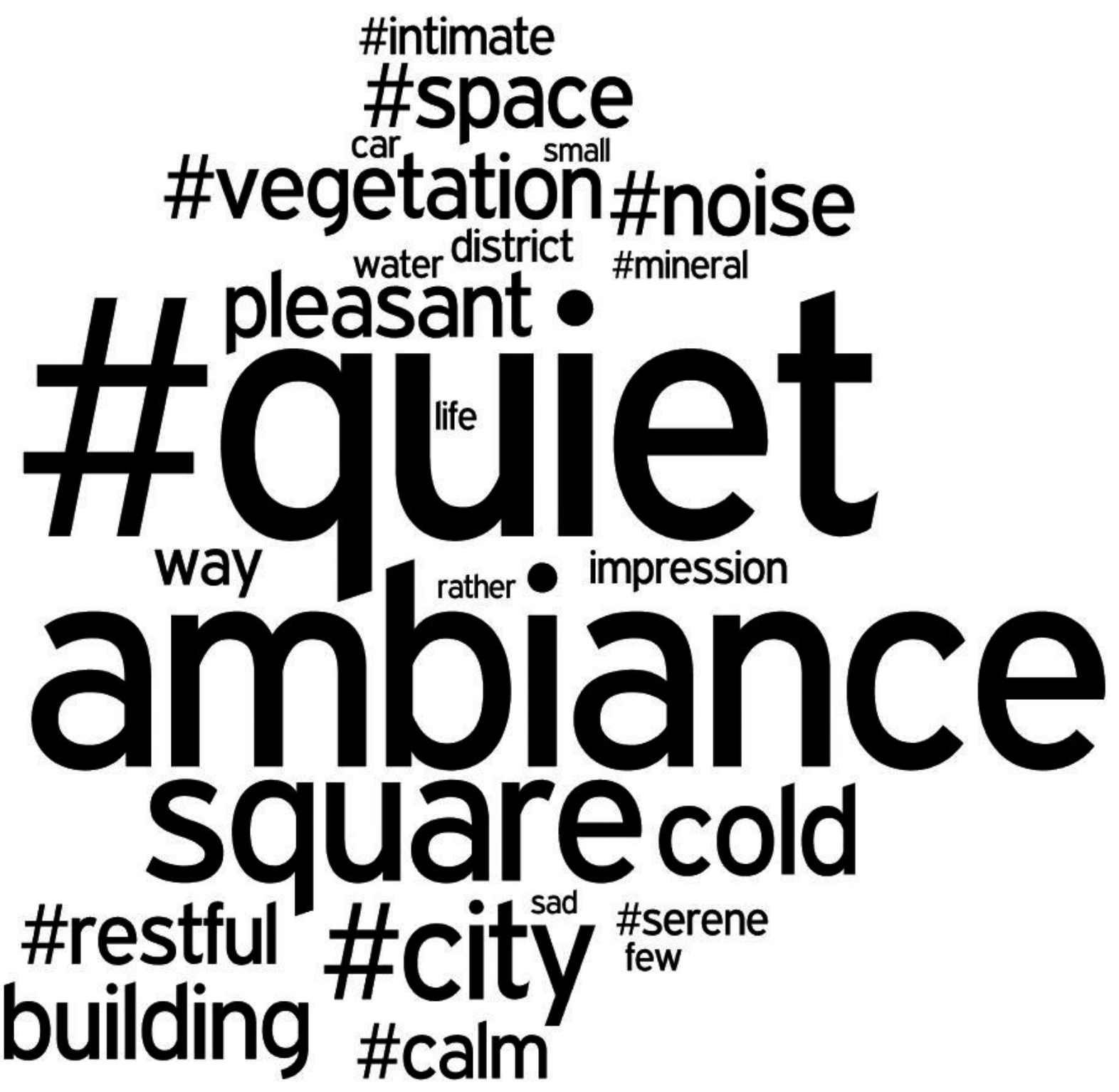

Figure 7: Tag cloud, occurrences of lemmas used by interviewees to answer the question 'How would you describe the ambiance of this square?' for the three squares (Tag cloud realised with Wordle). The size of the police of lemmas is proportional to their occurrence (number of times quoted by the interviewees). The position of the lemmas is purely aesthetic 
The interviewees were more attentive to the sonic ambiance of the squares in December than in September. According to D. Aubrée and S. Carbonnel (1983), the tones of places change with the rhythm of hours, days, seasons. According to authors, in research the seasonal temporality influences sonic perception in public space. For example, P. Amphoux (1996) talks about a 'warm silence' and about summer being 'more silent than winter', while W. Yang and J. Kang did not determine such differences. For the latter, although the seasonal variation must be taken into account in the evaluation of comfort, research did not show significant differences between the seasons in terms of acoustic evaluation (Yang \& Kang, 2005). Other authors (Griffiths, Langdon \& Swan, 1980; Vallet, 1996) have observed a seasonal effect on sonic annoyance. L. Yu and J. Kang, in their 2010 study about factors influencing sonic preference in exterior urban spaces, noted that seasonality only influences sound judgement for some sounds (sounds of water, human sounds and mechanical ones). Investigating seasonal temporality as a parameter which may potentially influence sonic perception in urban zones seemed to be a necessary line of research. It is important to take seasonality into account, including as a factor that influences other potentially explanatory variables in perceptive differentiation. Seasonality is particularly instructive, because it leads to variations in vegetation, transportation, the presence of birds, temperature and periods of sunshine and, therefore, practices which influence sonic perception in particular.

The lemma 'cold' is, indisputably, used more frequently in December than in September (11 occurrences in December against 2 in September). But we have to emphasise that this result depends on the geographical localisation of the fields of study (France in this context). The lemmas 'mineral', 'city' and 'open' are also more recurrent in December than in September. On the other hand, some terms are used in a more iterative way in September than in December: the lemmas 'vegetation' (due to seasonality), 'intimate', 'modern' and 'restful' in particular.

The terms most frequently quoted to qualify these three squares differ according to the qualified square. The analysis of the lemmas used by the interviewees to describe their impression of the squares reveals differences between the lemmas used in September and those used in December. The size of the square is quoted in a more recurring way during the first visit in September, while the intimate character of the square is prevailing among the interviewees during the visit in December. Spatiality is thus dominant during the discovery of the square, while the general impression of the place is associated with a feeling during the second visit.

Place Centrale is qualified by the terms 'open' (19 occurrences) and 'quiet' (14 occurrences). The urban form, the volumetric and the opening of a square engender a certain perception of the sonic ambiance. The link between the urban form and a feeling of protection in front of urban nuisances (particularly noise pollution) is expressed in particular by the interviewee 10AMM, who in the questionnaire 
explains a positive feeling: 'Impressive buildings, sensation to escape the nuisances of the city' (Interviewee 10AMM).

\section{Description in 5 words}

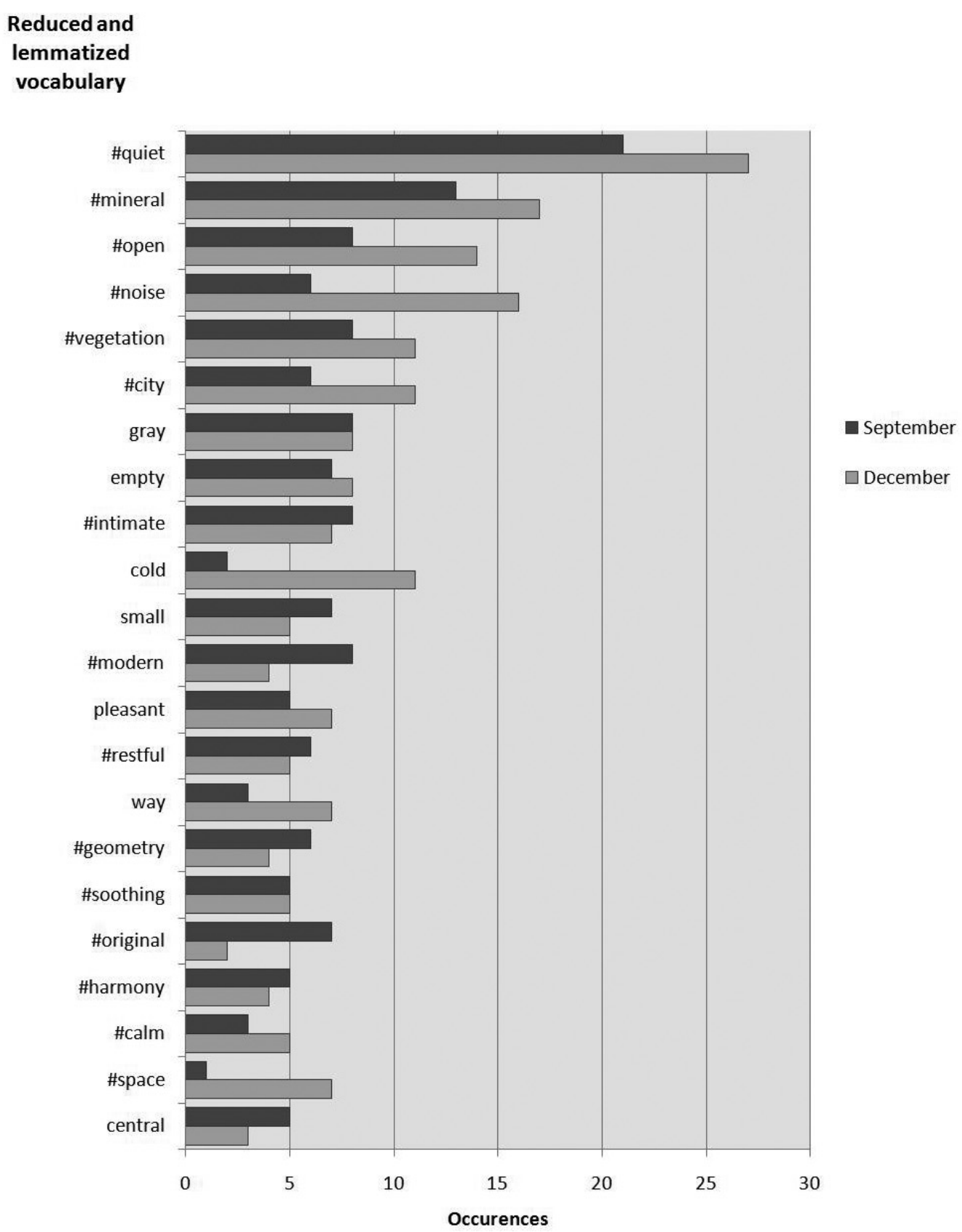

Figure 8: Occurrences of lemmatised vocabulary (with Sphinx Lexica Software) used by interviewees to describe the squares in five words, in both seasons 


\section{Description in 5 mots}

\section{Reduced and \\ lemmatized \\ vocabulary}

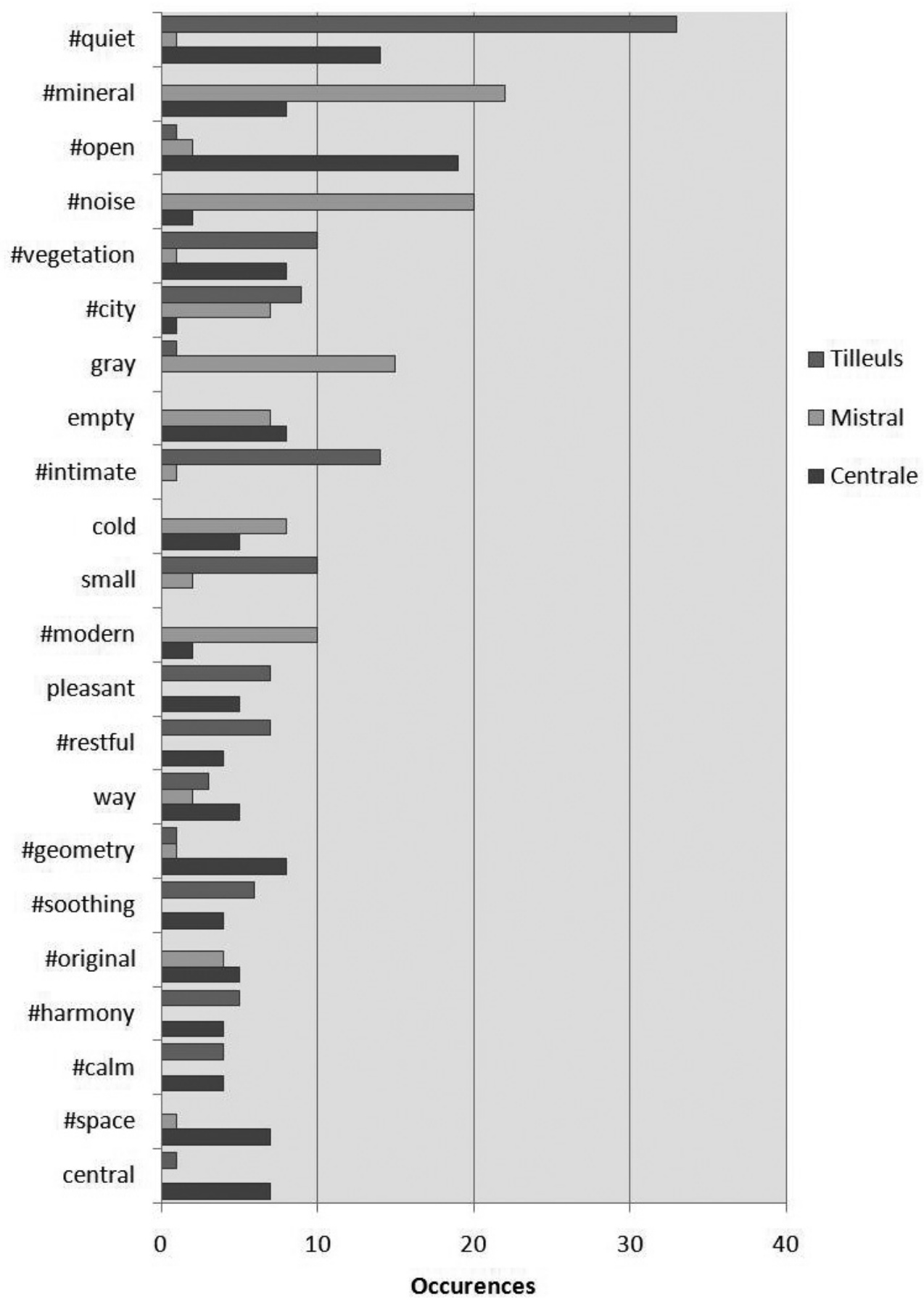

Figure 9: Occurrences of lemmatised vocabulary (with Sphinx Lexica Software) used by interviewees to describe the squares in five words, for each square 


\section{Sonic perception in public spaces}

Noise is the subject of a selective distortion mechanism of sound message perception. Hearing a 'noise' is to make a choice, a selection, in a completely subjective and personal way. According to E. Lecourt, 'our perception deals with filters' (2006). These filters do not only depend on the capacity of hearing; they also concern the familiarity maintained with certain sounds, the nature of a sound source that is or is not useful or not (according to the feature of the source), the signification given to the sound. Our ears are thus selective, and this selection depends both on collective and individual factors. The perceptual process is therefore a mechanism of information selection. This selection of perceptive information is possible, according to G. Fischer (1997), thanks to differentiations such as close/distant or important/common place.

L. Yu and J. Kang (2008) point out that some determining factors in sonic evaluation have been investigated in connection with housing environments. Urban public space has rarely been the object of research, except in particular in the CRESSON laboratory (Centre de recherche sur l'espace sonore et l'environnement urbain), which worked on common sonic ambiance in public spaces. Some research is based only on certain personal parameters (concerning reactions to traffic noises) such as age, sex, socioeconomic status or subjective sensibility to noise (Belojevic \& Jakovljevic, 1997). For example, R. Guski (1999) analyses, for people living in a noisy environment, the personal and social variables of annoyance caused by noise.

Analysing the ambiances consists of a method with which to approach the urban environment via everyday situations, in situ, implying physical elements, practices, perceptions and representations. The study of urban ambiances includes not only physical signals, spatio-temporality and perceptive aspects, but also individual and collective representation and social interaction. Sonic ambiance is intrinsically connected to the space; the public space is characterised by an ambiance which varies temporarily.

The figure 10 illustrates the significant relation between feelings and judgements of sonic ambiance, established on the basis of our analysis of these questionnaires. So, when the interviewee feels good, the sonic ambiance seems to him or her to be generally pleasant, and the sonic ambiance is considered unpleasant if the interviewee does not feel good. This implies an interrelation between sonic ambiance assessment and general feeling. In other words, the sonic environment has an influence on people's well-being in public squares.

Vegetation and space are the elements evoked by the interviewees to explain their positive feelings on the square (figure 11); on the other hand, noise is the most quoted element with which to clarify a negative feelFigure 11: Occurrences of lemmatised vocabulary (with Sphinx Lexica Software) used by interviewees to describe their feelings on the squares. 


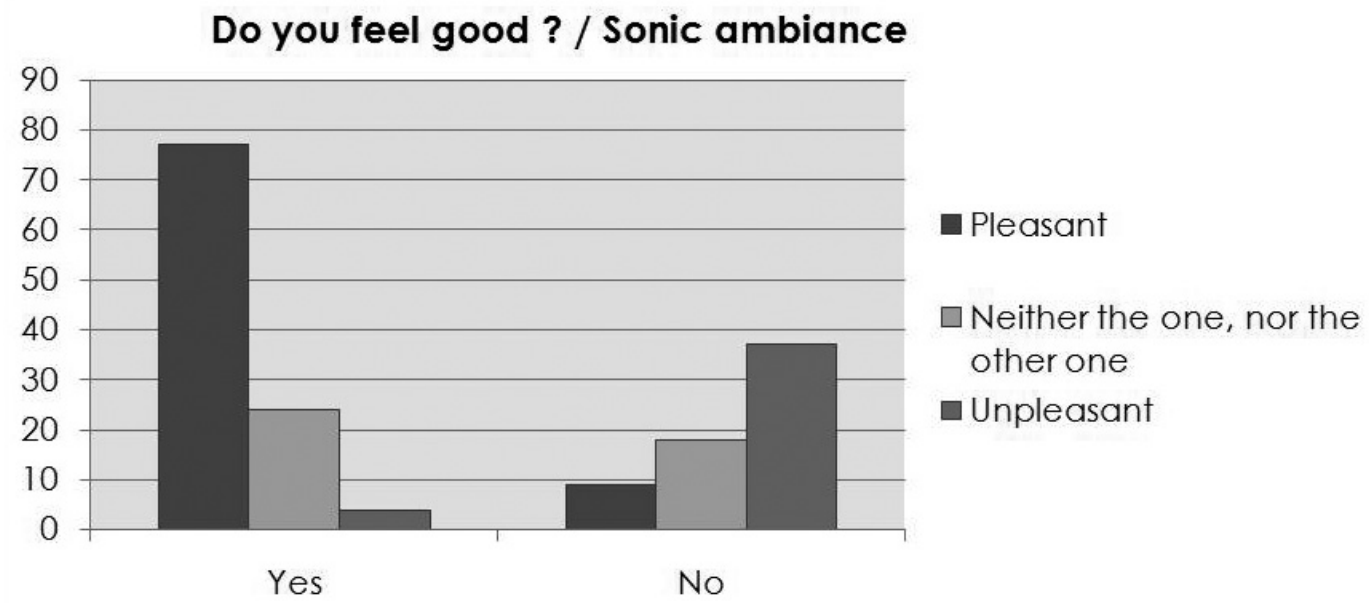

Figure 10: Crossed board of answers to the questions regarding sonic ambiance (pleasant, unpleasant, neither) and feelings (good or bad feelings); $p=<0.0 \% ; \mathrm{chi} 2=75,69 ; \mathrm{ddl}=2$ (VS). The relation is very significant

The sound sources (such as the means of transportation) are clearly more present on the pictures of the squares in December ( 45 occurrences) than in September (23 occurrences); sonic ambiance thus seems to be more influential to the interviewees' perception of the overall ambiance in December than in September.

Interviewee 9AMV comments on his picture of the Place Centrale in December (figure 12): 'in December, I wanted to show [...] the tramway behind, because it is true that we heard the tramway very much this time, more than in September I think, and this is a part of the environment of the square,. In the same sense, interviewee 30CRV notes a seasonal difference in the sonic perception: 'this was for the problem which appeared more during the second visit [in December]; it is because of the noise of the roadwork and the street'. Interviewee 8AMV associates the sound of the tramway with the overall ambiance of the place: 'and there we see the tramway, which is a part of the ambiance of the square'; while interviewee 10 AMM analyses the sonic presence of the tramway as a symbol of urbanity: 'later I took a photo of the tramway, because it was a source of noise, not necessarily a nuisance, but which reminded us that we were in a more or less urban site and not in the countryside'.

When a source of sound is experienced negatively, it can be masked. The most eloquent example is the masking work on freeways (sometimes, noise-reducing walls can have a dual function, acoustic and visual, has and thus have a dual impact on sonic perception).

During the individual interviews, we presented the interviewee with his photographs of the ambiance of each of the three squares, taken in both seasons, and asked him to comment on and clarify his choices. Then we asked him what was missing in his photographs to fully describe the overall ambiance of each square. The occurrences of used terms, presented in figure 15, indicate that, according to 


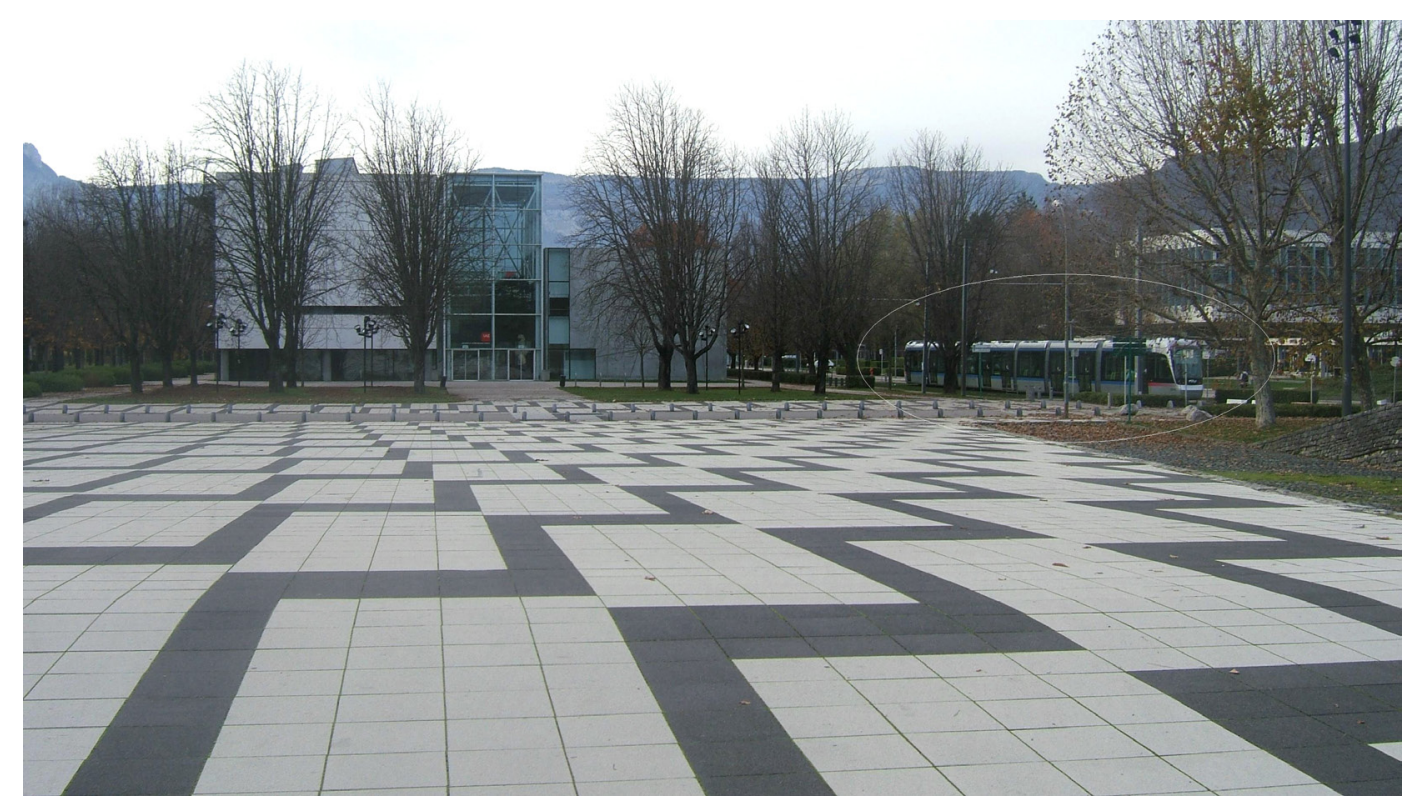

Figure 12: Photograph of Place Centrale ambiance, December (interviewee 9AMV)

the interviewees, for all the squares the sonic aspect is missing, when it comes to describing the ambiance of the squares. The lemmas 'noise' (mentioned by 20 interviewees), 'sound' (10 occurrences), 'silence' (6 occurrences) and 'to hear' (4 occurrences) are recurrent in the interviewees' answers to what the photographs do not show about the perceived ambiance. 'It would be interesting to have the sonic ambiances of these three squares', interviewee 25CRV explained, much in the same way as interviewee 5AMM: 'with a photograph, what I can't express is the ambiance of the square. For the ambiance of Place Mistral-Eaux-Claires, we need sound'. 'To describe the ambiance of the square, it would be good to have photographs with sound to show how it can be calming, aggressive', interviewee 20BAM explained. 'I remember the sounds', interviewee $27 \mathrm{CRV}$ said. Therefore, the sonic ambiance alone seems to be stored in memory and remembered only via the visual ambiance of these spaces (seeing their photographs taken in the squares). Interviewee $28 \mathrm{CRV}$ expressed the absence, in his photographs (figures 13 and 14), of sonic ambiance: 'So noise is missing. But that we can't photograph it!'

In the same way, when we asked interviewee 30CRV what was missing in his pictures to show the overall ambiance, he answered: "noise, noise or silence, there is no element to take a picture of it. Actually, there is nothing showing noise or silence'. As for interviewee 14BAM: 'I would add sound. The ambiance, the sound level, I mean'. 


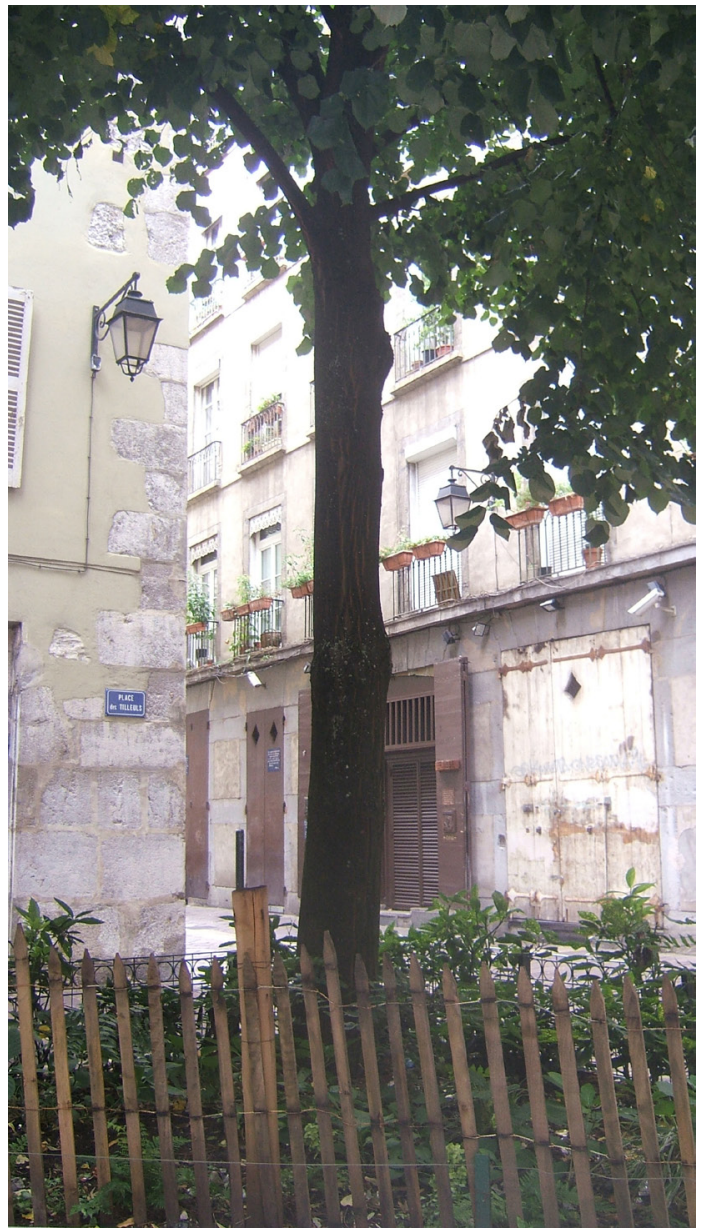

Figure 13: Photograph of Place des Tilleuls' ambiance, September (interviewee 28CRV)

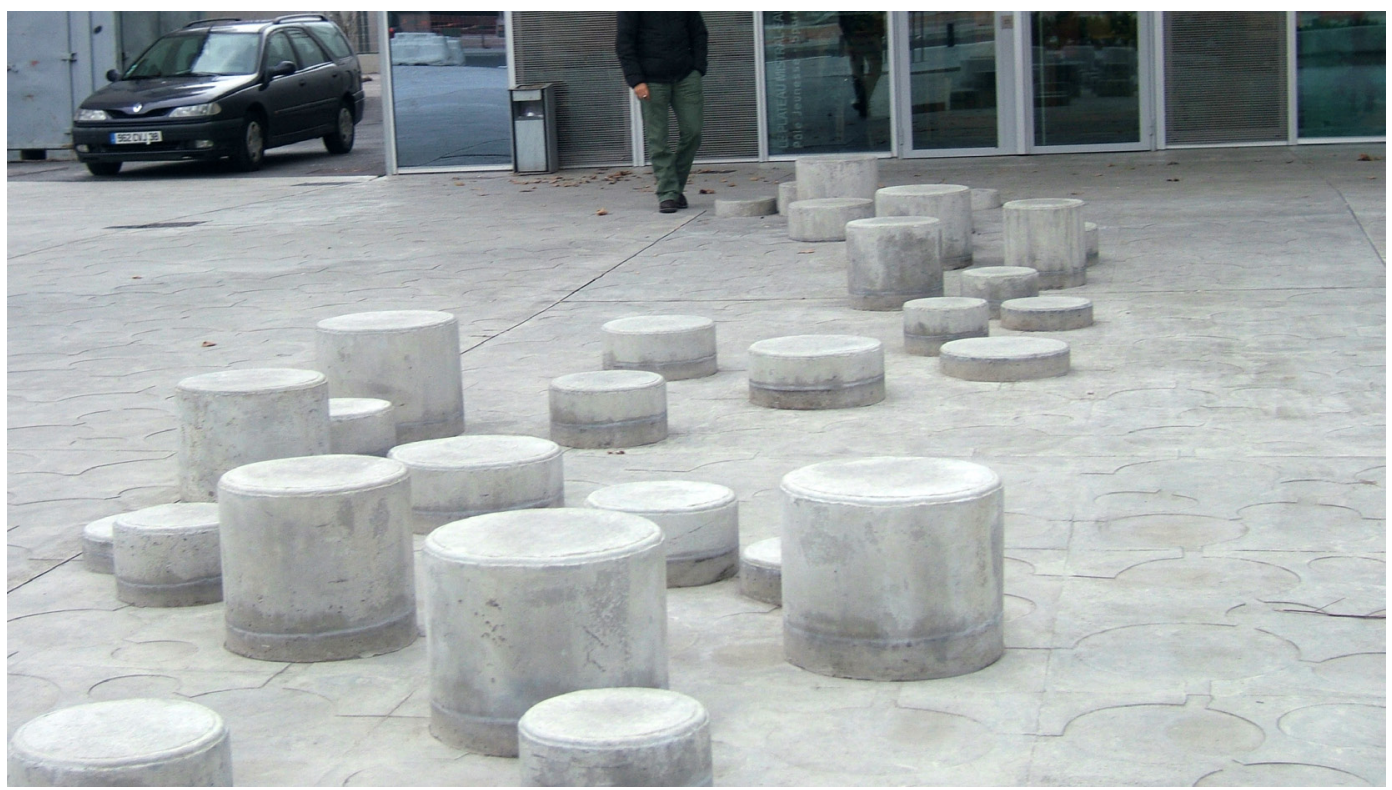

Figure 14: Photograph of Place des Tilleuls' ambiance, December (interviewee 28CRV) 


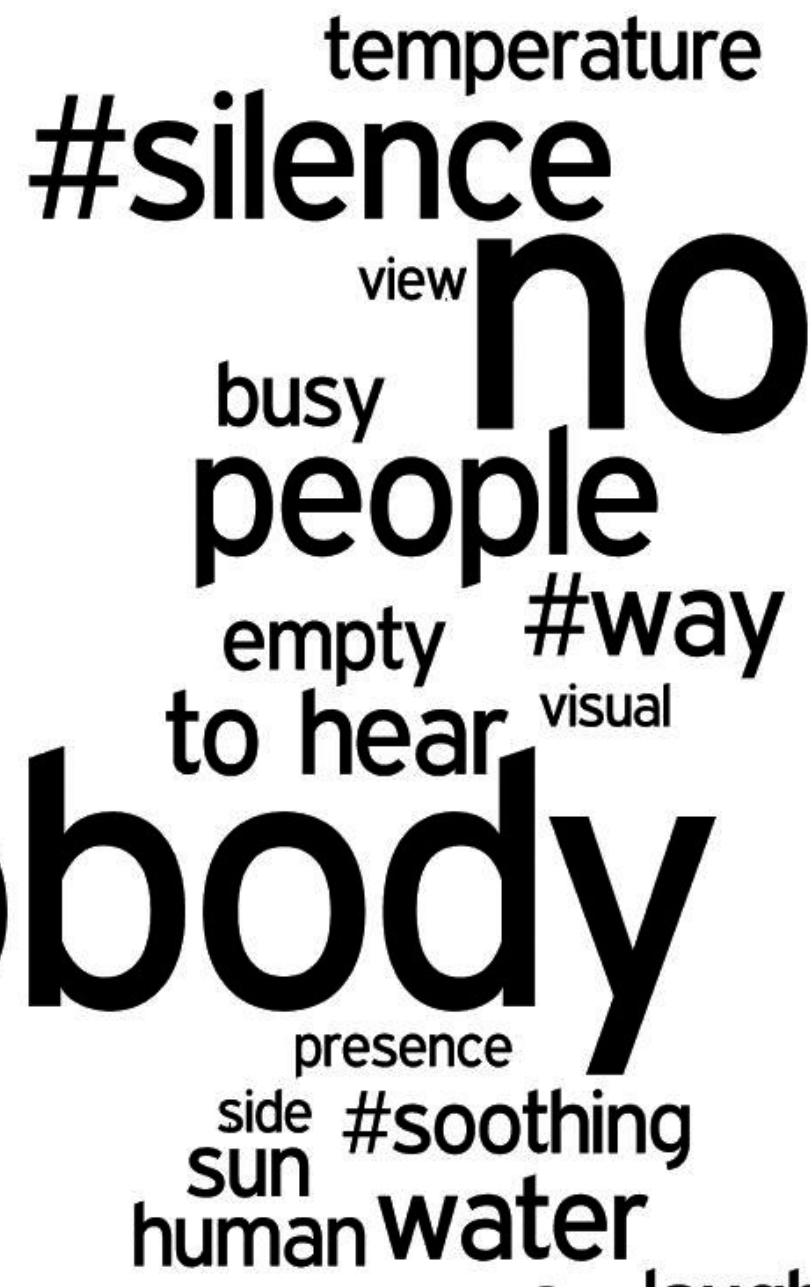

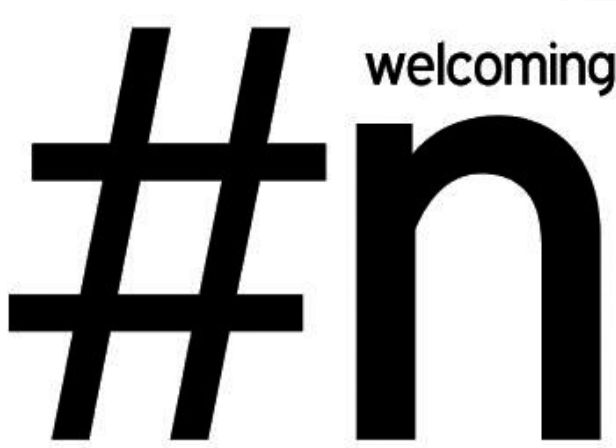

\#open landscape agressive laugh
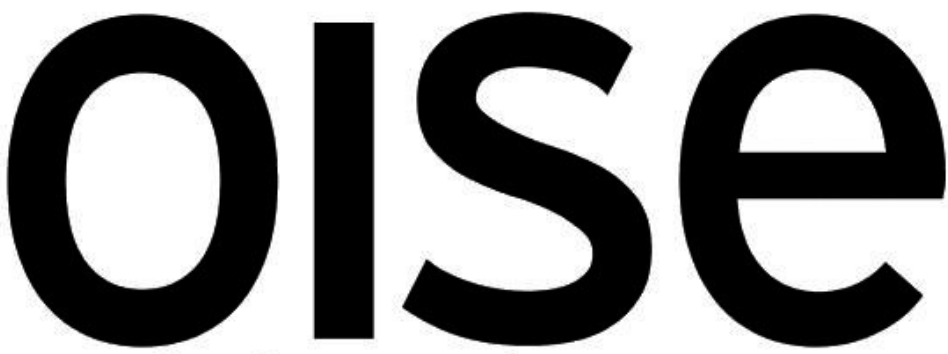

level

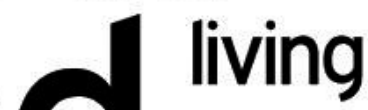
to speak road

Figure 15: Tag cloud. Occurrences of lemmatised reduced vocabulary with Sphinx Lexica software concerning the question 'What is missing in your photographs to fully describe the ambiance of the square?' (tag cloud realised with Wordle) 


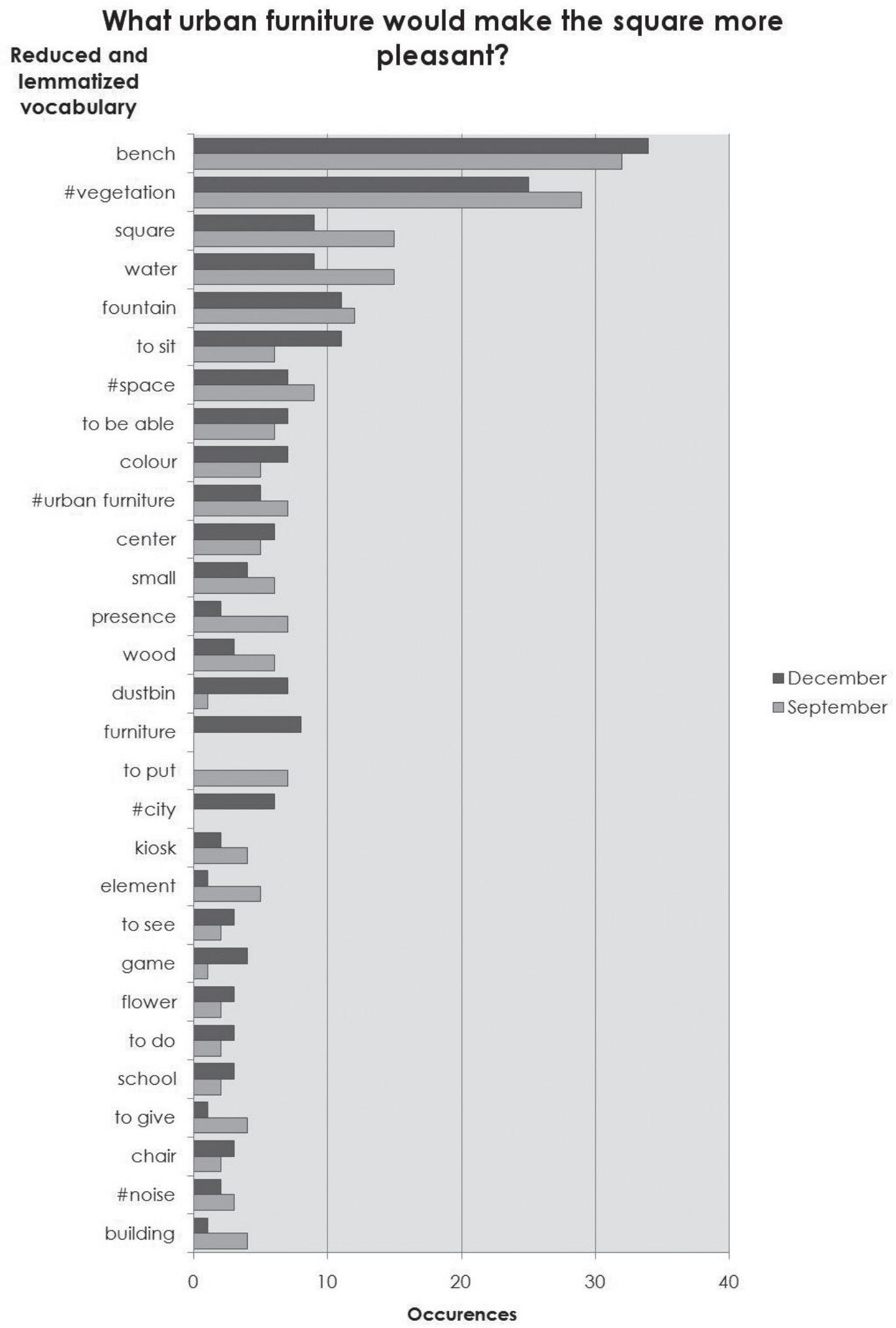

Figure 16: Occurrences of lemmatised vocabulary (with Sphinx Lexica Software) used by interviewees to answer the question 'What urban furniture would make the square more pleasant?' 
During the on-site questionnaire realised on each square, the interviewees had to indicate which urban fittings would make the square more pleasant. Figure 16 illustrates occurrences of lemmas used by the interviewees: benches and vegetation are the most quoted. C. Marquis-Favre, E. Premat and D. Aubrée (2005) remind us that $\mathrm{T}$. Yasmashita et al. have shown that a row of trees minimises expressed sonic annoyance. In the same way, A. Tamura (1997), to further diverse experiments, notes the influence of vegetation on people's feeling of annoyance. According to the experiment of J.-L. Carles, I. Lopez-Barrio and J. Vicente de Lucio (1999), the scenes in which sounds are most appreciated are the ones containing vegetation or a lot of water. Other studies (Milano, 1984; Pereira, 2003) have shown that the presence of vegetation affects environmental judgements (judged as more pleasant than spaces with no vegetation) and reduces the negative feelings caused by a high sonic level. B. Szeremeta and P.-H.-T. Zannin (2009) have thus shown, in their study of analyses and assessments of soundscapes in urban public spaces, that in the parks studied vegetation has a predominant influence on ambient sonic level perception. On the other hand, the presence of water is also mentioned as a necessity. The presence of water in an environment has been interpreted as an expression of qualities such as survival or fertility (Schafer, 1977). Furthermore, natural sounds and particularly water sounds generate a positive feeling of the environment (Carles, Lopez-Barrio, \& Vicente de Lucio, 1999). Water is also studied by P. Korosec-Serfaty (1982) in her study of representations associated with a square: she underlines the importance of water ('ornamental water', 'quiet water' and 'moving water') which has both an ornamental and a symbolic value. This presence of water is more often quoted by interviewees in September than in December. Seasonal impact must thus be underlined in this article. So should urban fittings be adjustable? The particularity of a public space is its temporal evolution and the diverse temporalities which give rhythm to it. The fixedness, which urban planners often search for erroneously, indeed does not correspond to the public space; on the contrary it is its temporal variability which makes it lively and shape these various urban times.

\section{Representations associated with sonic ambiance}

It seems necessary to consider the representations connected with the evaluation of urban sonic ambiances. Following the semi-directive individual interview, we used the method of so-called 'associative network', complementary of a discursive investigation method. Each interviewee was presented with a piece of paper with the words 'sonic ambiance of a square' written in the centre. The interviewee was then invited to write on the paper the terms he or she associated with this sentence, to number them and finally classify them as positive, neutral or negative. First, the analysis of lemmas associated with 'sonic ambiance of a square' (figure 17) reveals 

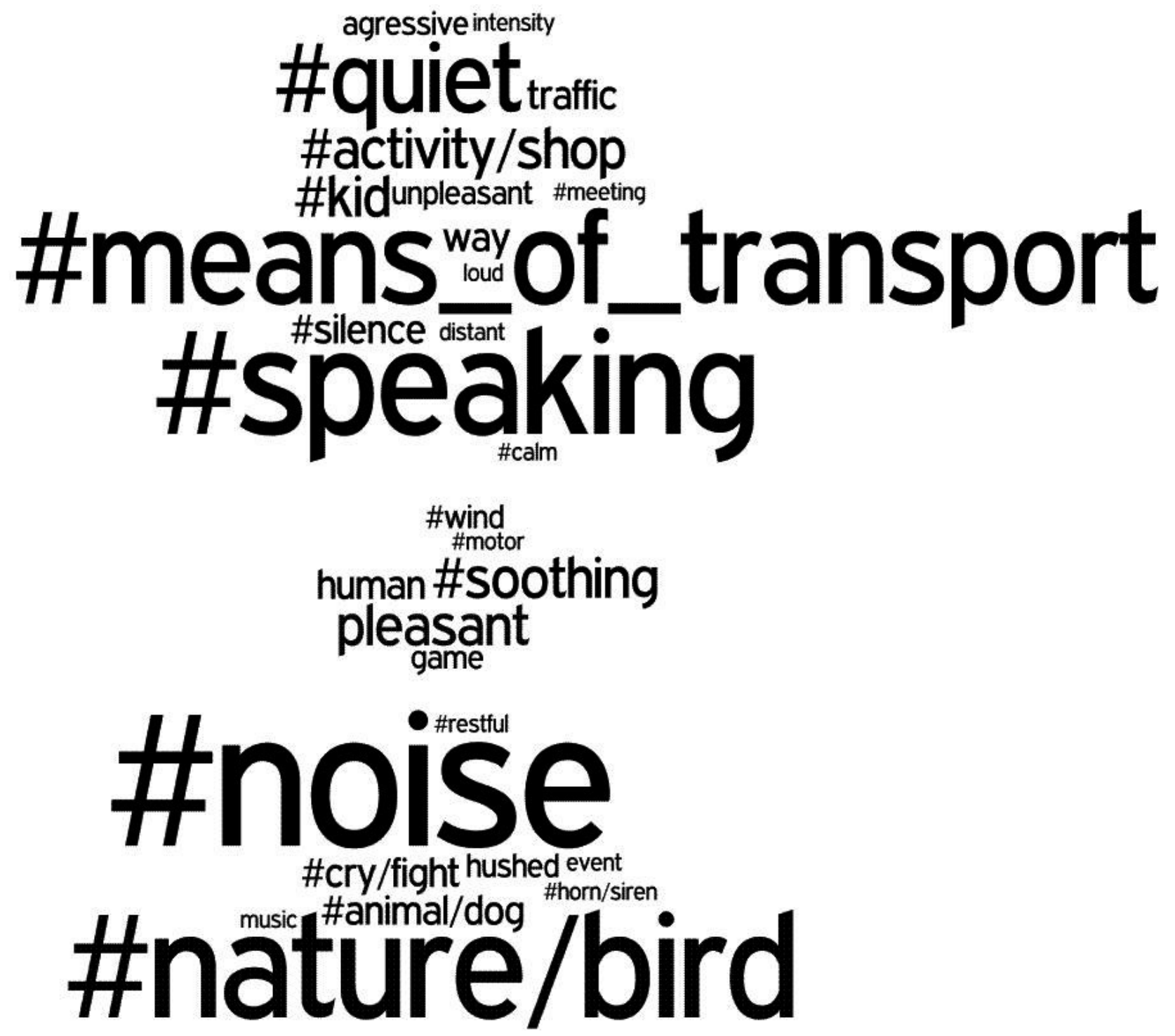

Figure 17: Tag cloud. Occurrences of lemmas associated by the interviewees with the sonic ambiance of a square (tag cloud realised with Wordle)

the omnipresence of terms associated with 'noise' (quoted by 23 interviewees out of 29), such as 'noise' and 'noisy'.

Second, the theme of oral expression ('speaking') - lemmas such as 'singing', 'voice', 'laughs, 'word', 'communication', 'verbal', 'discussion', 'shout' - is quoted by 18 interviewees. Oral expression and the grouping 'nature/bird' are the two second-most mentioned themes (18 occurrences). The evocation of transportation lemmas such as 'car', 'tramway', 'bus', 'transport', 'public transportation', 'plane' - is 

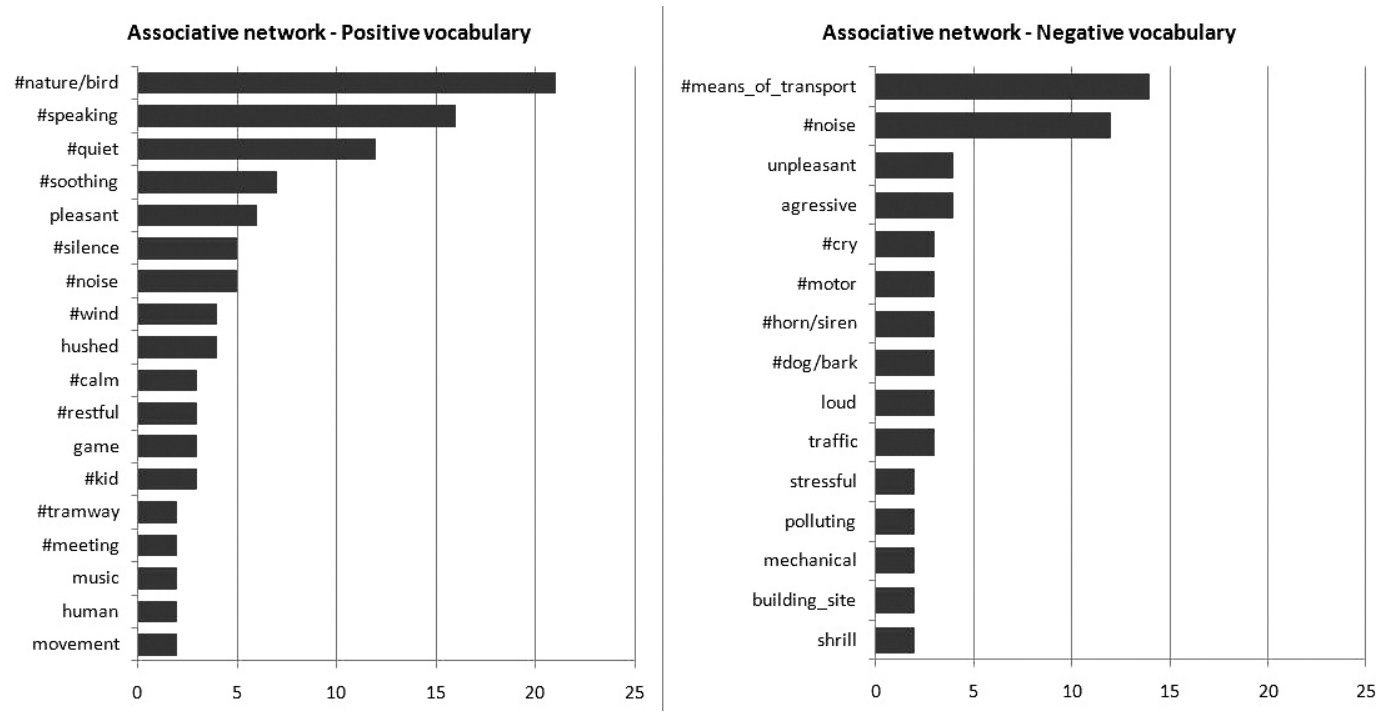

Figure 18: Occurrences of lemmatised vocabulary (lemmatised with Sphinx Lexica software) associated with the sonic ambiance of a square (associative network methodology)

present in the associative network of 16 interviewees. The lemma 'quiet' is stipulated by 12 interviewees; it is the fifth-most quoted term. The imagination associated with sonic ambiance therefore integrates, at the same time, the term 'noise' and, to a lesser extent, the term 'quiet'.

The positive lemmas associated with the sonic ambiance of a square are, most of the time (21 occurrences out of 29 associative networks), related to nature (figure 18), whereas the negative lemmas never deal with nature. Nature is clearly associated with the sonic ambiance of a public space, in a positive way. Interviewees' representations thus associate a pleasant sonic ambiance with nature. As for the negative lemmas present in the associative networks, they are concerned with transportation for 14 interviewees out of 29. The negative image of the sonic ambiance of a public square is clearly associated (for the largest number of occurrences) with sound sources such as transport. This sonic ambiance seems more influenced by sound sources located outside the square. The second most frequent occurrence of negative terms concerns the lemma 'noise', present in 12 associative networks. The term 'noise' is more often defined as a negative term (12 occurrences) than as a positive one (five occurrences) by the interviewees; the negative connotation associated with this terminology is revealed in the sonic imagination of people interviewed.

At the end of the individual in-depth interview, seven urban typologies were shown to the interviewee who had to rank them from one to seven according to quality environmental sounds: one for the most pleasant sonic environment and seven for the most unpleasant one. These seven typologies are: 
- Isolated individual house

- Private housing estate (pavillons)

- City small individual housing

- City medium collective housing

- Haussmannian housing ${ }^{1}$

- Big buildings

- Collective housing areas

I wanted to reveal the sonic representations associated with specific urban typologies; the objective was to compare these urban sonic imaginations with sonic perceptions and spatial and sonic evaluations of previous in situ investigations. We wondered if the sonic imagination associated with an urban typology had an impact on the perception and evaluation of a public space. On the other hand, if the type of housing environment, as a residential choice (except when it is forced), conditioned the sonic representations associated with urban typologies. The typology of the individual house is clearly associated by the interviewees with a pleasant sonic environment, although the gap between attributed marks is important, which shows that there is no consensus. It was indeed well-specified that the classification concerned the judgement (from positive to negative) of the sonic environment and not the sonic level. It also seemed necessary to highlight the fact that the sonic environment of an individual house in a private housing estate (pavilions) is estimated in a much more negative way than a small detached house in town and small/medium collective housing in town (figure 19). This result emphasises the fact that the individual or collective nature of a housing environment does not determine the evaluation and judgement of sonic environments associated with urban typology.

The evaluation of the sonic environment for each of the seven urban typologies presented to the interviewees (figure 20) shows that the isolated individual house is associated with a pleasant sonic environment by the interviewees in Group A, who live in individual houses themselves, more so than by the interviewees in the other groups: almost the entire group associated this typology with a pleasant sonic environment (scores from 1 to 3 ). The individual house in a private housing estate is also associated with a positive sonic environment by the residents in individual houses. City small individual housing does not get a higher score than 4 , and this goes for all groups. We can thus say here that regardless of the type of housing environment, this individual urban typology is never linked, in mind, with an unpleasant sonic environment, although its localisation in urban zones is specified. Let us note here that the individual or collective nature seems to be more clearly influential than the urban or outer-urban nature (or rural) in the evaluation of the associated sonic environment. The sonic environment associated with the typology of the small or 


\section{Urban forms - Sonic ambiance evaluation}

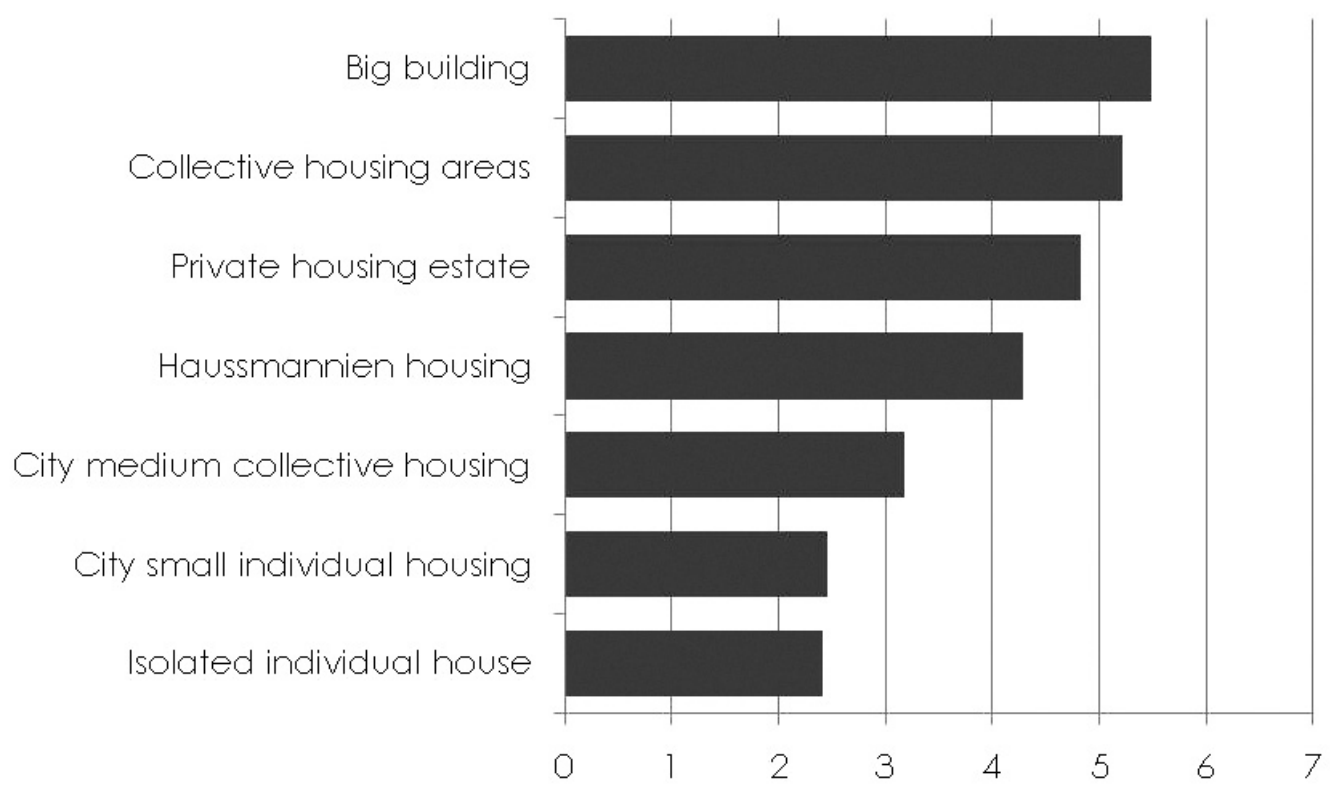

Figure 19: Sonic environment associated with urban typologies (1: more pleasant, 7: more unpleasant)

medium collective housing in town is, paradoxically, judged more positively than the individual house in a private housing estate, even by interviewees who live in an individual house (marked from 1 to 4 for the entire Group A). As the type of housing is a choice for all the interviewees, we can draw a parallel between the choice of housing environment and the sonic representations associated with urban typologies. Haussmann housing is to a greater extent associated with an unpleasant sonic environment by Group C, interviewees who live in recent collective housing. This urban typology, associated in mind with the old central districts, does not seem to correspond to the interviewees' - those who choose to live in a recent housing typology - desired sonic environment. To a certain extent, big buildings seem to be associated more with an unpleasant sonic environment than collective housing by people living in an individual house or in recent collective housing, and vice versa for people living in old collective housing.

The urban typologies are thus associated, in the imagination of city dwellers, with various sonic environments, considered more or less pleasant, not only with regard to the sound level, but also to the types of sounds heard and associated with urban and sonic ambiances.

This study has enabled us to distinguish differences in sonic imagination associated with urban typologies according to housing typology. Our residential choices 
Isolated individual house

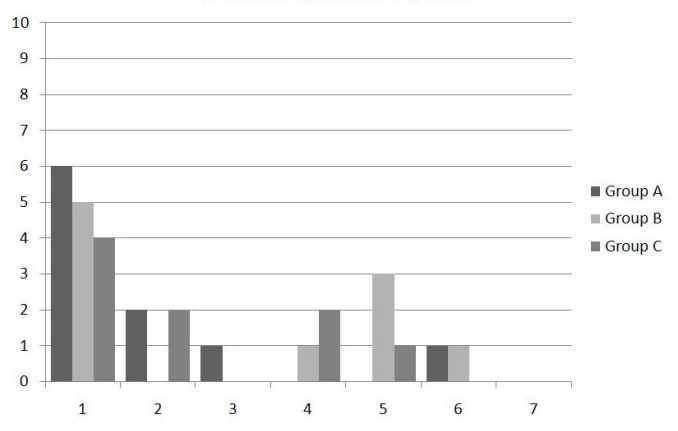

Private housing estate

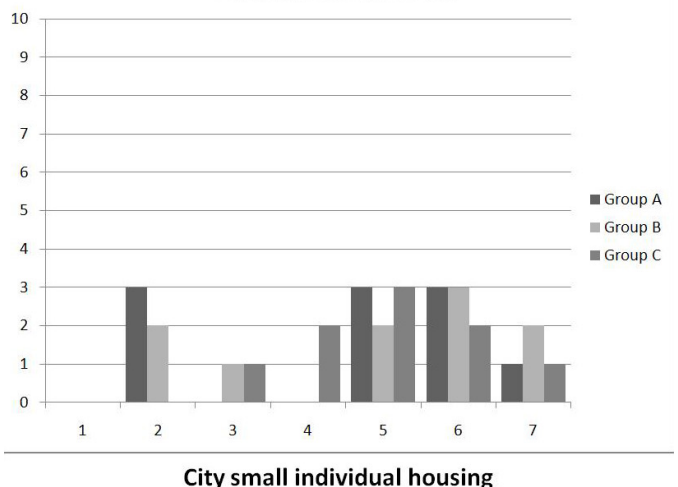

City small individual housing

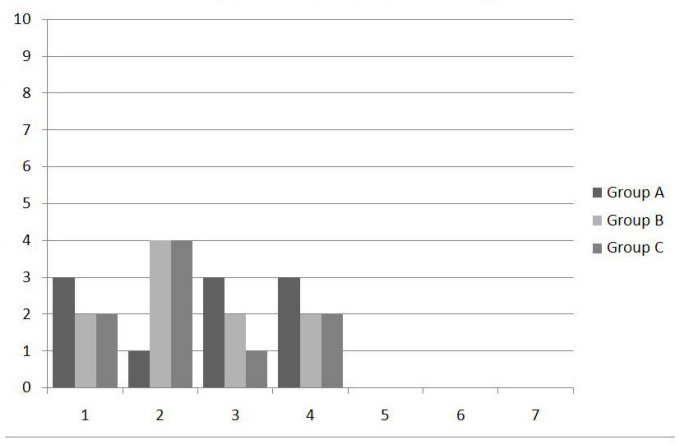

City medium collective housing

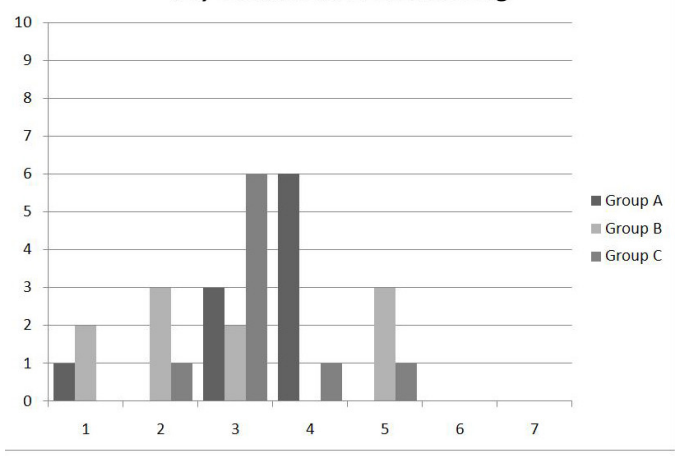

Haussmannien housing

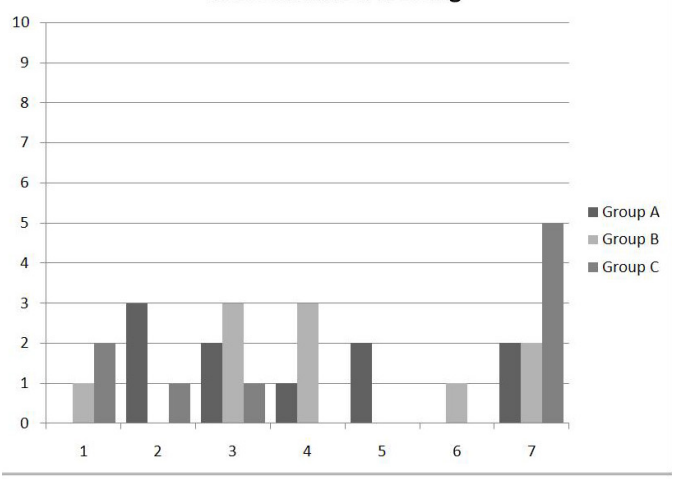

Big building

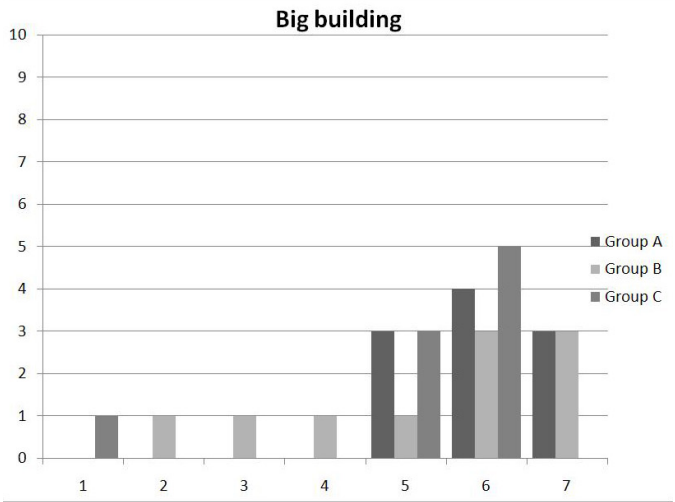

Collective housing areas

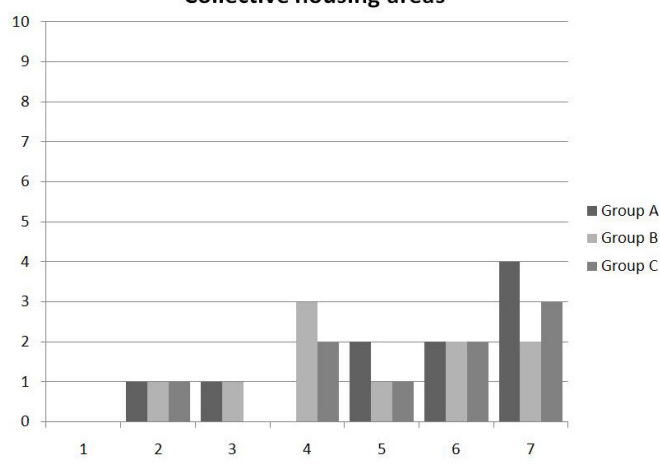

Figure 20: Assessment of sonic environment associated with seven urban forms.

Group A: interviewees living in individual houses Group B: interviewees living in old collective housing Group C: interviewees living in recent collective housing 
are thus guided by representations associated with typologies. Does this sonic imagination not condition, in turn, the sonic perception of spaces and urban forms?

\section{Conclusion}

To conclude, the methodological protocol elaborated enabled us not only to reveal some parameters influencing sonic perception in urban public spaces during in situ evaluations, but also to understand sonic representations associated with a number of urban typologies. We could show that some terms linked to sonic environments were used by the people interviewed to describe the ambiance of different urban spaces. The seasons parameter seems to stand out in the investigations; the three squares studied are qualified as more quiet in September than in December. A very significant relationship has also been established, further to the analysis of the questionnaires, between feeling and judgement of sonic ambiance.

Contrary to vegetation and space which, according to the interviewees, characterise their positive feeling of public squares, noise is linked to a negative feeling. We have seen that on the photographs taken by interviewees to describe the ambiance of the squares, sonic sources have been shown consciously, and most interviewees indicate that sounds are missing in the photographs to completely describe the ambiances perceived. The associative network method shows that the sonic ambiance of a square is associated by most of the interviewees with lemmas linked to sounds, but also, to a lesser extent, to oral expression and nature.

Finally, we studied sonic representations associated with urban typologies. The individual house typology is clearly associated by the interviewees with a pleasant sonic environment, even if we saw that the individual or collective nature of housing is not decisive in the assessment and judgement of sonic environments associated with urban typologies.

\section{Notes}

1. Georges-Eugène Haussmann (1809-1891) was a French civic planner whose name is associated with the rebuilding of Paris and a specific housing typology.

\section{References}

Amphoux, P. (Eds.). (1996). Au seuil de l'audible. Cresson.

Aubrée, D., \& Carbonnel, S. (Eds.). (1983). Indices sonores et représentations. CSTB.

Belojevic, G., \& Jakovljevic, B. (1997). Subjective reactions to traffic noise with regard to some personality traits. Environment International, 23(2), 221-226.

Carles, J., Lopez Barrio, I., \& Vicente de Lucio, L. (1999). Sound influence on landscape values. Landscape and Urban Planning, (43), 191-200. 
Fischer, G. (Ed.). (1997). Psychologie de l'environnement social (2nd ed.). Dunod.

Griffiths, I., Langdon, F., \& Swan, M. (1980). Subjective effects of traffic noise exposure: reliability and seasonal effects. Journal of Sound and Vibration, 71(2), 227-240.

Guski, R. (1999). Personal and social variables as co-determinants of noise annoyance. Noise \& Health, 1(3), 45-56.

Korosec-Serfaty, P. (1982). Images de la place. Étude des représentations des formes, fonctions, pratiques et modes de la sociabilité des places publiques. Ministère de l'environnement et du cadre de vie.

Lecourt, E. (Ed.). (2006). Le sonore et la figurabilité. L'Harmattan.

Leobon, A. (Ed.). (1986). Analyse psycho-acoustique du paysage sonore urbain. Strasbourg, Université Louis Pasteur.

Lopez Barrio, I., \& Carles, J. (1995). Acoustic dimensions of inhabited areas: Quality criteria. The Soundscape Newsletter, (10), 6-8.

Lopez, F. Schizophonia vs l'Objet Sonore: Soundscapes and Artistic Freedom. Accessed on: http:// www.franciscolopez.net/schizo.html

Marquis-Favre, C., Premat, E., \& Aubrée, D. (2005). Noise and its effects - A review on qualitative aspects of sound. Part II: Noise and annoyance. Acta Acustica, 91(4), 626-642.

Milano, M. (Ed.). (1984). Evaluation and analysis of street arborization in Curitiba.

Moser, G., \& Weiss, K. (Eds.). (2003). Espaces de vie. Armand Colin.

Pereira, M. (2003). Noise perception in the public space: indicators of noise tolerance in the city of Rio de Janeiro. In: Curitiba, Brazil, 779-786.

Schafer, R.M. (Ed.). (1979). Le paysage sonore, toute l'histoire de notre environnement sonore à travers les âges. MandM. JC Lattès.

Schafer, R.M. (Ed.). (1977). The tuning of the world. McClelland and Stewart. Toronto.

Southworth, M. (1969). The sonic environment of the cities. Environment and behaviour, (1), 49-70.

Szeremeta, B., \& Trombetta Zannin, P. (2009). Analysis and evaluation of soundscapes in public parks through interviews and measurement of noise. Science of the Total Environment, (407), 6143-6149.

Tamura A. (1997). Effects of landscaping on the feeling of annoyance of a place. In: Proceedings of symposium on psychological acoustics (135-161).

Vallet, M. (Ed.). (1996). Caractéristiques et indicateurs de la gêne due au bruit des avions.

Yang, W., \& Kang, J. (2005). Soundscape and sound preferences in urban squares. J Urban Des, (10), 69-88.

Yu, L., \& Kang, J. (2008). Effects of social, demographical and behavioral factors on the sound level evaluation in urban open spaces. JASA, 123(2), 772-783.

Yu, L., \& Kang, J. (2010). Factors influencing the sound preference in urban open spaces. Applied Acoustics, 71(7), 622-633. 